\title{
Desenvolvimento e aplicação de um objeto de aprendizagem m-learning para crianças da educação infantil
}

\section{Development and application of an m-learning object for children in preschool education}

\section{Nathalie Suelen do Amaral Gonçalves}

E-mail: nathaliegoncalves@unipampa.edu.br

\section{Érico Marcelo Hoff do Amaral \\ E-mail: ericoamaral@unipampa.edu.br}

\section{RESUMO}

Este trabalho visa inferir se a utilização de tecnologia da informação como instrumento de apoio ao ensino-aprendizagem pode influenciar e ajudar na identificação de formas geométricas básicas e ligeira indicação das cores primárias na educação infantil. Foi proposto e desenvolvido um jogo para tablet (objeto de aprendizagem), aplicado e observado em turmas do maternal e jardim de infância. Neste artigo são descritas as etapas da implementação, a descrição das interfaces a fim de identificar a adequação desta proposta, que como resultado considerou-se adequado para as crianças da turma do jardim de infância, mostrando verbalmente que fixaram o conteúdo proposto.

Palavras-chave: Jogo, objeto de aprendizagem, formas geométricas, educação infantil.

Abstract This work aims to infer whether the use of information technology as an instrument to support teaching-learning can influence and help in the identification of basic geometric shapes and a slight indication of primary colors in early childhood education. A tablet game (learning object) was proposed and developed, applied and observed in nursery and kindergarten classes. This article describes the stages of implementation, the description of the interfaces in order to identify the adequacy of this proposal, which as a result was considered suitable for children in the kindergarten class, showing verbally that they fixed the proposed content.

Keywords: Game, learning object, geometric forms, preschool education.

\section{Introdução}

Atualmente, as crianças nascem, crescem, e ao seu redor, equipamentos eletrônicos conectados à internet disponibilizam todo o tipo de interatividade. 
Desde muito novas são instigadas a interagir, tanto da forma lúdica como tecnológica. Esta última, para elas, é muito mais natural do que foi para seus pais. Segundo Dias apud Prensky (2015), nativo digital representa a geração de crianças nascidas a partir do século XXI que possuem domínio nato e fazem uso da tecnologia digital com extrema facilidade. Diferente dos imigrantes digitais, que são indivíduos que não necessariamente nasceram neste meio (da tecnologia), mas ao longo de sua vida foram aprendendo formas de utilizar e interagir com ela (PESCADOR, 2010).

Os indivíduos atualmente estão inseridos em um meio em que a tecnologia se faz presente das mais variadas formas no nosso cotidiano, principalmente como elemento construtivo de nossas reflexões e atividades. Segundo Francisco (2015), não seria diferente com as crianças, onde muitas delas têm incluídas na sua rotina equipamentos eletrônicos, principalmente o tablet, sendo cada vez mais comuns e presentes em quase todos os ambientes que as crianças frequentam, como escola, casa de amigos, ou até mesmo a sua residência. Sendo assim, a tecnologia impacta de forma direta na vida e desenvolvimento da criança, desde muito cedo, como por exemplo, na educação infantil.

Considerando o contexto exposto acima, propõe-se a aplicação de uma ferramenta como instrumento para verificar o conhecimento das crianças na faixa etária de 2 a 4 anos, de uma escola particular de Educação Infantil, localizada na cidade de Uruguaiana/RS, no que diz respeito à identificação de formas geométricas básicas e cores primárias, através de um jogo executado no tablet.

\section{EDUCAÇÃO INFANTIL E AS BRINCADEIRAS}

Quando se reflete superficialmente em brincadeiras, e no ato de brincar das crianças, somos remetidos à atividade inata o qual elas estão acostumadas, pois já é de sua natureza. Segundo Wajskop (1995), estudos mostram que a valorização da infância pode ter iniciado baseada em uma concepção idealista e protetora da criança. Brinquedos eram utilizados na recreação, e a proposta era voltada para os sentidos das crianças. Esta autora aponta que ao longo do tempo, houve uma mudança na educação que costumava ser de forma tradicionalista e verbal.

A Educação Infantil surgiu com uma proposta de educação diferenciada, focada nas características das crianças de acordo com sua faixa etária, sendo 
voltada ao sensorial, utilizando-se de jogos e materiais didáticos adequados ao período de desenvolvimento da criança. Para chegar a este patamar, a Educação Infantil foi foco de estudos e sofreu influência de diferentes estudiosos e seus respectivos pensamentos e filosofia. Podem ser citados alguns desses estudiosos conforme aponta Wajskop: Fröbel, Maria Montessori e Décroly.

Por fim, diante de todos os argumentos, se infere que "a brincadeira é uma situação privilegiada de aprendizagem infantil" (Wajskop, 1995, p. 67). A autora também fala que a brincadeira passou a ser considerada um espaço de experimentação e estabilização de conhecimentos e afetos, por meio de interações entre crianças e adultos.

\section{TECNOLOGIAS APLICADAS A EDUCAÇÃO INFANTIL}

Vive-se em uma sociedade contemporânea permeada pelas tecnologias e, com isso temos acesso rápido a essas ferramentas utilizando-as com uma frequência cada vez mais intensa. As escolas de educação infantil, bem como, as famílias precisam adaptar-se e aprender a utilizar essas ferramentas tecnológicas que desde muito cedo as crianças estão sendo estimuladas e expostas a elas cotidianamente.

Nesse intuito pensamos em uma proposta de jogo interativo criado com o objetivo de servir como objeto de aprendizagem, do tipo m-learning, que, de acordo com Franciscato e Medina (2008), trata-se de uma variação do e-learning que é a aprendizagem eletrônica, geralmente realiza através de computadores, na modalidade à distância.

O m-learning é uma variação caracterizada por ocorrer através de dispositivos móveis, ou mobile. Citam-se como exemplo os smartphones e tablets, tendo como vantagem possibilitar acesso aos conteúdos e interações de qualquer local e a qualquer momento. Segundo Scaico et al. (2012), o m-learning promove a ubiquidade no processo de ensino aprendizagem, e esta característica favorece a aprendizagem e assimilação. Além das ferramentas citadas anteriormente, salienta-se que propiciar um ambiente dinâmico e motivador vai qualificar e intensificar o processo de construção da aprendizagem nas crianças.

Para que o potencial aprendizado seja atingido ao máximo, Franciscato (2008, p. 3) indica diz que para "a construção de soluções de aprendizagem com mobilidade, é necessário que estas devam priorizar os critérios de usabilidade, 
acessibilidade, mobilidade, colaboração/cooperação". Assim como também conceitua os objetos de aprendizagem como "qualquer entidade, digital ou não digital, que possa ser utilizado, reutilizado ou referenciado durante o aprendizado suportado por tecnologias".

\section{ASPECTOS RELACIONADOS À APRENDIZAGEM NA FAIXA ETÁRIA DE 2 A 4 ANOS}

A criança constrói conhecimentos a partir dos estímulos apresentados ao seu redor, no ambiente em que vive, suas interações e ações nortearão seus pensamentos, que conduzirá seu desenvolvimento (ARAÚJO, 2009). O autor ainda aborda sobre as características das crianças da educação infantil a partir dos dois anos de idade, mostrando que estas características impactam diretamente no propósito deste trabalho pois, o objeto educacional só será adequado à turma se ela estiver dentro das capacidades das crianças na faixa etária da turma.

As capacidades e aquisições motoras e cognitivas das crianças podem ser separadas em dois grupos: de dois até três anos e de três anos até quatro anos. Não foi encontrado na pesquisa informações de que crianças do primeiro grupo possam reconhecer ou copiar formas geométricas básicas. Crianças do segundo grupo podem ter essa capacidade de reconhecer ou copiar formas geométricas básicas. As crianças do primeiro grupo têm como características principais motoras e cognitivas: manipular objetos com as mãos, pode reproduzir um círculo sobre papel ou areia, memória e capacidade de concentração aumentam. Para o segundo grupo, tem-se como características: copiar figuras geométricas simples, desenha uma pessoa de forma simples, reconhece três cores, começa a ter noção das relações de causa e efeito, é bastante curiosa e observadora (FERREIRA, 2013).

O brincar e os jogos são instrumentos primordiais para o desenvolvimento pleno da criança, sendo assim é possível utilizar as ferramentas tecnológicas para instigar e estimular o uso de jogos para trabalhar as características de cada faixa etária e suas respectivas habilidades cognitivas, motora, afetiva e social. Como estudo, sugeriu-se um jogo que envolva a motricidade fina, cores, formas e as figuras geométricas básicas para que a criança relacione uma coluna com a outra de mesma categoria. 


\section{TRABALHOS CORRELATOS}

Segundo Pereira et al., (2012), os objetos de aprendizagem por meio do lúdico é um recurso que promove a interatividade, permitindo a reflexão do conteúdo apresentado. Pereira em seu artigo que possui o título: "O uso da tecnologia na educação, priorizando a tecnologia móvel”, aborda sobre o uso de jogos como objeto de aprendizagem. Os objetos de aprendizagem em formato de jogos são indicados e utilizados para auxiliar no processo de ensinoaprendizagem, tendo muito potencial e importância na transmissão de conhecimento e novos valores. Porém, estes precisam ser bem planejados para que a ludicidade evidencie os conhecimentos apresentados. Eles tiveram como objetivo informar sobre as duas potências em benefício de uma educação mais eficaz: jogos aplicados no ensino e as tendências tecnológicas. Na conclusão do trabalho, entre outras informações, foi mencionado que atividades lúdicas são fundamentais no processo de ensino, pois despertam o senso crítico, espírito construtivo, a imaginação e, sobretudo, o desejo de resolver as situações problemas.

Abech et al., (2012), em seu artigo "Um modelo de adaptação de Objetos de Aprendizagem com foco em dispositivos móveis", propõe atentar para o perfil e contexto de cada aluno, com objetivo de apresentar um conteúdo mais adequado às necessidades educacionais de cada estudante, proporcionando um outro modo de desenvolver seu aprendizado através de sistemas móveis. Isto se torna possível quando se implementa um ambiente que pode adaptar objetos de aprendizagem de acordo com o perfil dos alunos, de modo que tais implementações foram avaliadas através de um cenário de uso. Os resultados demonstraram a viabilidade de uso do modelo, bem como destacaram a adaptação dos objetos de acordo com alguns contextos.

Outra pesquisa relevante a ser discutida nesta seção é denominado "Desenvolvimento de Jogo Educacional sobre Ecotoxicologia Utilizando HTML5", de Bispo et al., (2012, p.1), que teve como objetivo auxiliar no ensino de conceitos de toxicologia ambiental para os alunos de graduação. Para tanto, foi desenvolvido um jogo educativo para ensinar o ciclo de vida e o uso de microcrustáceos do gênero Daphnia. Foi utilizado o HTML 5, assim como na presente pesquisa, para o desenvolvimento do objeto de aprendizagem, e com o 
trabalho foi possível concluir que a linguagem é adequada para o determinado fim, além de ser uma plataforma livre, aberta e padronizada internacionalmente. Também neste mesmo estudo, foi aplicado parte do jogo (inicial) em crianças da pré-escola, e foi possível concluir que o uso do objeto de aprendizagem em formato de jogo nestas crianças em idade pré-escolar obteve resultados promissores e um grande interesse pela atividade.

\section{MATERIAIS E MÉTODOS}

A metodologia de pesquisa aplicada a este trabalho é qualiquantitativa, que segundo Almeida (2015), a pesquisa quantitativa considera opiniões e números e faz tradução em informações que serão analisadas e classificadas, bem como de forma qualitativa, que considera a relação entre mundo e sujeito, sendo descritiva, onde o autor tende a analisar os dados indutivamente.

É possível afirmar que esta pesquisa também é realizada por método indutivo de observação, que considera o conhecimento como baseado na experiência. A generalização deriva de observações de caso da realidade e são elaborados a partir de constatações particulares, de acordo com Almeida (2015).

O presente trabalho foi realizado inicialmente com um estudo sobre as características motoras e cognitivas de crianças dentro da faixa etária citada, sobre a educação infantil e a questão do uso das brincadeiras como complemento de ensino visando aprendizado de formas geométricas básicas. Pesquisou-se também sobre as tecnologias mais utilizadas pelas crianças, onde se encontrou tablet como dispositivo móvel mais amplamente utilizado, por isso a aplicação do objeto de aprendizagem foi utilizado neste equipamento em específico.

Inicialmente buscou-se a linguagem de programação ideal para desenvolver o jogo, onde foi tentada a utilização da linguagem de programação Java. Porém, não foi possível completar o desenvolvimento diante de dificuldades técnicas encontradas. Destas dificuldades, pode-se citar diretamente a lentidão de processamento da compilação. Isto ocorre, pois, para mostrar a execução do que foi realizado, o computador precisa trabalhar em conjunto com um emulador ${ }^{1}$ (Android Virtual Device Manager) no ambiente de desenvolvimento Eclipse. O conhecimento limitado em programação na linguagem Java também influenciou

\footnotetext{
${ }^{1}$ Emulador é um sistema de computação que reproduz funções de outro sistema operacional, permitindo executar softwares sobre ele.
} 
para que fosse pesquisada outra linguagem de programação.

Durante o desenvolvimento, foi estudado sobre interface, usabilidade e heurísticas para que o jogo se torne adequado para as crianças e que seu aprendizado seja potencializado pelo uso deste recurso, complementando as demais formas de ensino em sala de aula.

Após desenvolvido e testado foi aplicado para dois grupos de alunos. $\mathrm{O}$ grupo 1 que faz parte da turma do Maternal, com 6 alunos, onde o objeto de aprendizagem foi aplicado individualmente, duas vezes em cada criança, na faixa etária de 2 a 3 anos. $O$ grupo 2 faz parte da turma do Jardim, com igualmente 6 alunos, na faixa etária de 3 anos a 4 anos. Sendo assim, terá por finalidade a análise da capacidade de identificação de formas geométricas básicas (quadrado, círculo e triângulo).

Este jogo é inspirado em brinquedos já existentes, lúdicos, que de acordo com Pereira et al., (2012), aprendizado lúdico é aquele que ocorre por meio de jogos educativos, em que a criança aprende brincando, onde também bem observa (através da citação de Dewey - 1952, pensador norte-americano,) que "o jogo faz parte do ambiente natural da criança, ao passo que referências abstratas e remotas não correspondem ao interesse da criança".

O objeto de aprendizagem foi desenvolvido em 2017 na linguagem HTML5 (HyperText Markup Language - versão 5) juntamente com a linguagem Javascript, com framework JQuery², principalmente utilizados JQueryUI e JQuery TouchPunch, que são bibliotecas para criação de elementos de interface de usuário e adaptação para tela touch dos dispositivos móveis. O CSS3 (Cascading Style Sheets - versão 3) é a linguagem utilizada para adicionar estilo para página onde será apresentado o jogo.

O jogo implementado pode ser executado em qualquer sistema operacional, visto que utiliza a tecnologia de interpretação, nativa de navegadores WEB. Contudo, os experimentos para validação da aplicação foram realizados em um tablet, rodando a plataforma Android, que tem sua natureza como código aberto, sendo amplamente difundido entre os usuários.

Alinhado com as ideias de Bispo et al., (2012), esta solução pode ser

2 JQuery é uma biblioteca de funções Javascript, que interage com HTML, desenvolvida para facilitar a programação e execução de scripts. 
utilizada por diferentes arquiteturas, deste que conectadas à Internet, sendo assim acessível, não necessitando de nenhuma instalação específica no dispositivo móvel para que execute, funcionando também em desktops.

Ele é compatível com a maioria dos navegadores atuais. O recomendado é o Google Chrome atualizado na versão a partir da 58.0.3029.110.

Figura 1. Jogo das Formas sendo executado no navegador Google Chrome

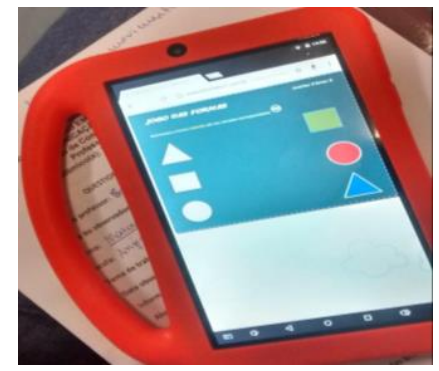

Figura 2. Interface do jogo, acerto do encaixe e erro do encaixe (respectivamente)
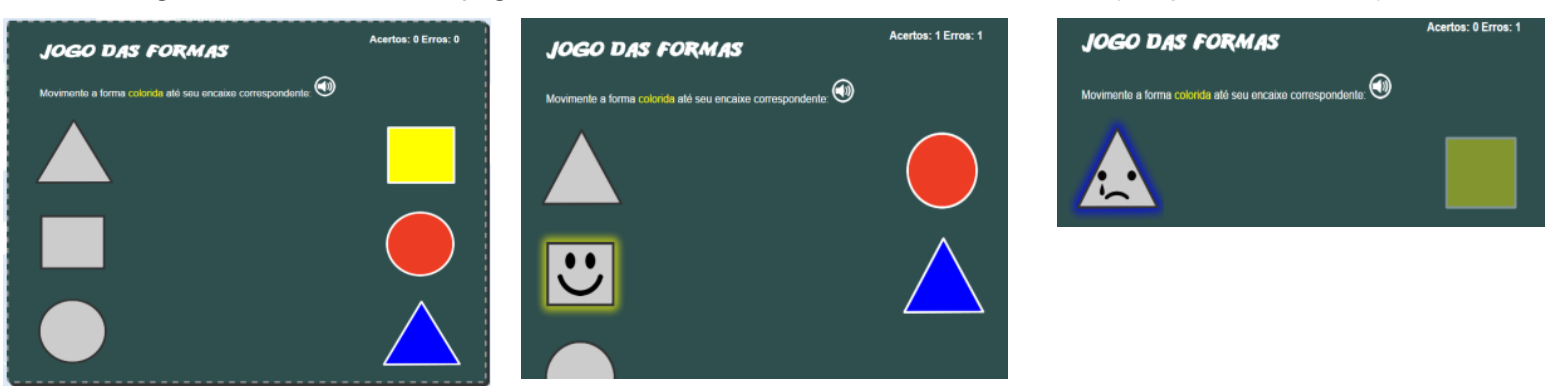

Foram criados os desenhos que fazem parte do jogo e também o layout da tela em si, conforme demonstrado na Figura 2. O layout foi programado na tela através do arquivo estilo.css. O arquivo principal do jogo é o teste.html, conforme pode ser observado na figura 5. Nele, está contido também o código javascript que dá funcionamento ao jogo. Há também outros arquivos de bibliotecas que fazem parte do projeto, são eles: jquery-1.7.2.min.js, jquery-ui.min e jquery.ui.touch-punch.min.js.

O jogo consiste em três formas geométricas básicas: quadrado (amarelo), círculo (vermelho) e triângulo (azul). Eles são pintados com as três cores primárias, utilizadas justamente para estimular a aprendizagem também destes elementos. As formas estão situadas na vertical à direita. À esquerda estão os encaixes de cada forma. O objetivo é que o aluno arraste com dedo a forma colorida e a encaixe no seu local correspondente. Ao realizar os três encaixes de maneira correta o jogo acaba e pode ser reiniciado.

As dez heurísticas de usabilidade de Nielsen foram estudadas para que a interface 
do objeto de aprendizagem fosse o mais adequada possível ao público-alvo. (ANDRADE, 2007).

O principal instrumento utilizado foi a observação participante. A observação direta de cada aluno individualmente e também suas interações em grupo permitiu inferir várias questões que serão abordadas mais adiante. Houve o acompanhamento da professora, mas o observador foi o mesmo aplicador do objeto. Este procedimento permite obter elementos de muitas áreas de desenvolvimento e atividades que podem ser ou não adequadas e interessantes para aquelas crianças. Também podemos dizer que é de grande valia, pois é realizada no contexto natural da sala de aula, junto do educador (TAVARES, 2015).

\section{RESULTADOS E DISCUSSÃO}

A partir da aplicação do objeto de aprendizagem e observação da atuação de cada um dos alunos, foi possível inferir que o jogo é adequado para o grupo de crianças de 3 a 4 anos (turma do jardim de infância), e ainda não adequado para o grupo de crianças de 2 a 3 anos (turma do maternal).

O grupo de crianças na faixa etária de 2 a 3 anos (maternal), não conseguiu executar a atividade de forma satisfatória, pois, mostraram muita dificuldade de arrastar a forma até seu encaixe. De seis crianças, apenas uma conseguiu conduzir as formas de modo satisfatório. As demais tocavam com várias partes da mão na tela, fazendo com que não fosse possível selecionar a forma e fazer a atividade (pouca motricidade fina, conforme observa-se na Figura 03). Outra dificuldade encontrada foi que eles levavam a forma para além da área do jogo. Isto poderia ter sido previsto através da heurística Prevenção de Erros, se tivesse sido programado um limite para movimentação dos elementos apenas dentro da área do jogo. Metade dos alunos entenderam e acertaram em colocar as formas no encaixe correto, mas claramente notou-se que eles não entendem que é uma forma geométrica. Eles entendem como um desenho que deve ser levado ao seu semelhante. Todas as crianças esboçaram reações de emoção quanto aos feedbacks audiovisuais, ficando ligeiramente tristes quando erravam e muito felizes quando do acerto. 
Figura 3. Gráfico quantitativo de alunos do maternal

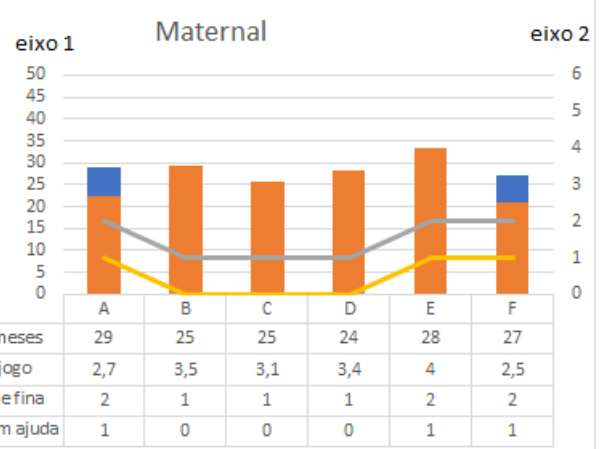

Figura 4. Gráfico quantitativo de alunos do jardim de infância

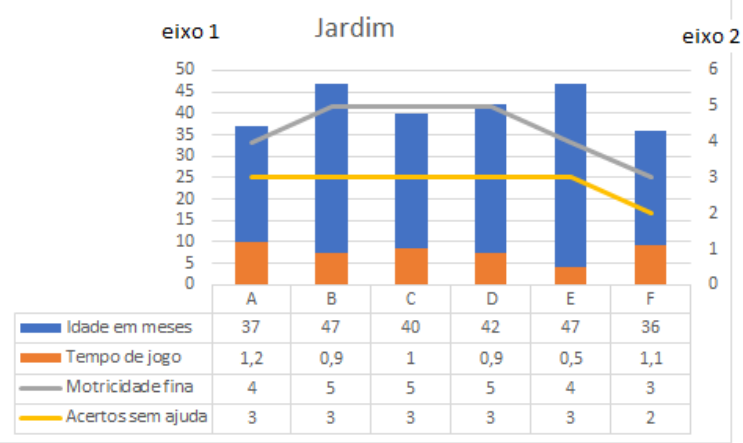

Ainda neste grupo, foi perguntado à professora se já tinha sido abordado e mostrado sobre as formas geométricas básicas, o que foi respondido de que ainda não, pretende-se iniciar no segundo semestre. Outras brincadeiras lúdicas com as formas também não haviam ainda sido apresentadas.

O tempo de atividade de jogo de cada aluno do maternal foi em torno de 3 minutos, todos necessitaram de auxílio do aplicador para completar o jogo. Muitas tentativas com erros e dificuldades com a motricidade fina, em selecionar e arrastar a forma até o local correto. Claramente as instruções do jogo mesmo no formato de áudio não foram suficientes para o entendimento do jogo, que precisou ser explicado com muita calma individualmente para cada aluno.

No grupo de crianças na faixa etária de 3 anos a 4 anos (jardim de infância), houve cem por cento de aproveitamento na realização da atividade, conseguiram completar o jogo de forma satisfatória.

Os alunos deste grupo mostraram muito entusiasmo na realização da atividade. Dos seis alunos, dois apresentaram pouca dificuldade, não na resolução dos encaixes em si, mas sim na parte tátil da interação (motricidade fina), conforme apresentado na Figura 04. A professora informou que estes alunos não costumam ter contato com o tablet, ao contrário dos demais alunos que já estão acostumados.

Todos os alunos da turma do jardim de infância conseguiram completar o desafio na primeira vez de jogar. Mostravam interesse em repetir verbalmente 0 
nome da forma e a cor (conforme o áudio informava), enquanto arrastavam a forma até seu encaixe. Este grupo claramente compreendeu o propósito do jogo, assimilando como são as três formas mostradas, indicando as três cores primárias, e realizando o encaixe de forma correta. Em média os alunos desta turma levaram 1 minuto cada um para jogar as duas vezes. Para todos a explicação via áudio foi suficiente. Muitos deles nem precisaram de explicação, tateando a tela já perceberam que as formas coloridas se movimentavam e instintivamente levaram às suas semelhantes, recebendo o feedback de acerto, e, posteriormente, completando o jogo de forma muito rápida.

Foi perguntado para a professora deste grupo se já havia sido abordado sobre as formas e mostradas aos alunos. Ela respondeu que apenas superficialmente, e que será tratado de forma mais específica e com brincadeiras no segundo semestre do ano letivo.

\section{CONCLUSÃO}

Ao longo da aplicação do objeto de aprendizagem nos alunos das turmas de Maternal e Jardim de Infância, foi possível inferir que caso tivesse sido realizada uma entrevista com os educadores das turmas previamente, talvez fosse desenvolvido um objeto de aprendizagem adequado para ambas as turmas.

No entanto, o objeto de aprendizagem não foi considerado adequado para o maternal, pois as crianças ainda não têm a motricidade fina bem desenvolvida, apresentando muitas dificuldades para manusear o equipamento com tela sensível ao toque. Outro fator é que ainda não compreendem os conceitos de formas básicas e cores. Por fim, seu entendimento ficou apenas na identificação de figuras semelhantes umas com as outras.

O grupo do jardim de infância compreendeu a atividade apresentada, sendo para este grupo, o objeto de aprendizagem considerado adequado e de resultados amplamente satisfatórios, conseguindo todos, ao final da atividade, identificar corretamente e verbalizar cada uma das formas e suas respectivas cores, e seus encaixes correspondentes.

Espera-se que esta pesquisa possa contribuir com as discussões em torno do assunto, e ao final, é possível concluir que sim, a utilização de tecnologias da informação e comunicação e objetos de aprendizagem na educação infantil tem grande influência, quando utilizada de forma complementar aos demais recursos 
pedagógicos; e que os objetos de aprendizagem, quando aplicados ao públicoalvo certo e de forma diferenciada das atividades do cotidiano, exercem grande motivação e interesse nos alunos. Sendo assim, eles conseguem assimilar de maneira mais descontraída o conteúdo proposto.

\section{Referências}

Abech, Márcia; Da Costa, Cristiano André; Barbosa, Jorge; Rigo, Sandro; Cambruzzi, Wagner. Um Modelo de Adaptação de Objetos de Aprendizagem com foco em Dispositivos Móveis. Rio de Janeiro - RJ, 2012. Disponível em; <https://goo.gl/Jt3xoA >. Acesso em: 9 jan. 2017.

Almeida, Maurício B. Noções básicas sobre metodologia de pesquisa científica. Belo Horizonte - MG. Universidade Federal de Minas Gerais, DTGI-ECI, 2015. Disponível em: <https://goo.gl/niYV2N>. Acesso em: 29 mai. 2017.

Araújo, R. M. B. Características das Crianças em cada Faixa Etária. 2009. Disponível em: <https://goo.gl/uXdfp6 >. Acesso em: 14 abr. 2017.

Bispo, Diogo Moreira; Zabeu, Márcio Silvatti; Umbuzeiro, Gisela Aragão; Borges, Marcos Augusto Francisco. Desenvolvimento de Jogo Educacional sobre Ecotoxicologia Utilizando HTML5. Campinas - SP, Revista Brasileira de Informática na Educação, v. 20, n. 1, 2012. Disponível em: <https://goo.gl/u7RfiS >. Acesso em: 30 abr. 2017.

Brasil. Ministério da Educação e do Desporto. Secretaria de Educação Fundamental. Referencial curricular nacional para a educação infantil / Ministério da Educação e do Desporto, Secretaria de Educação Fundamental. - Brasília: MEC/SEF, 1998. Volume1.

Dias, Ana Luisa Antunes. A criança Nativa Digital no Contexto de Filmes Publicitários. 2015. 121 f. Tese (Doutorado em Psicologia). - Faculdade de Ciências e Letras, Universidade Estadual Paulista "Júlio de Mesquita Filho", Assis, 2015.

Ferreira, H. C. S. Desenvolvimento infantil: O brincar e o aprender no préoperatório. São Luis - MA: Faculdade Santa Terezinha-CEST, 2013. Disponível em: <https://goo.gl/dMiFUU>. Acesso em: 14 abr. 2017.

Franciscato, Fábio Teixeira; Medina, Roseclea Duarte. M-Learning e Android: um novo paradigma? Porto Alegre - RS: CINTED-UFRGS, 2008. Disponível em: $<$ https://goo.gl/4N8XzN>. Acesso em: 9 jan. 2017.

Francisco, D. J.; Silva A. P. L. Criança e apropriação tecnológica: um estudo de caso mediado pelo uso do computador e do tablet. Maceió - AL. Universidade Federal de Alagoas, 2015. Disponível em: <https://goo.gl/8h24iV>. Acesso em: 29 mai. 2017.

Pereira, Leonardo R.; Schuhmacher, Vera Rejane; Schuhmacher, Elcio; Dalfovo, 
Oscar. O uso da tecnologia na educação, priorizando a tecnologia móvel. Blumenau - SC. 2012. Disponível em: <encurtador.com.br/chGLM>. Acesso em: 9 jan. 2017.

Pescador, C. M. Tecnologias digitais e ações de aprendizagem dos nativos digitais. V Congresso Internacional de Filosofia e Educação. Caxias do Sul - RS. Maio 2010. Disponível em: <https://goo.gl/gpd9QD>. Acesso em 15 abr. 2017.

ANDRADE, ANTONIO. Usabilidade de interfaces web: avaliação heurística no jornalismo on-line. Editora E-papers, 2007.

Scaico, Pasqualine Dantas; Lopes Diego; Azevedo, Max André; Silva, Jarbele C. da; Mendes Neto, Sinval V.; Falcão, Emmanuel de Souza. Implementação de um Jogo Sério para o Ensino de Programação para Alunos do Ensino Médio Baseado em mlearning. Rio Tinto - PB: Universidade Federal da Paraíba, 2012. Disponível em: <https://goo.gl/KjStQf >. Acesso em: 9 jan. 2017.

Tavares, Sabrina D. O brincar como meio de aprendizagem no jardim-de-infância. Porto - Portugal, 2015. Escola Superior de Educação Paula Frassinetti. Disponível em: <https://goo.gl/o7KQCd>. Acesso em: 29 mai. 2017.

Wajskop, Gisela. O brincar na educação infantil. São Paulo - SP: Cad. Pesq. oㅡ 92, p. 62-69, fev. 1995. Disponível em: <https://goo.gl/N6YG8r>. Acesso 